\title{
Étude sur modèle physique du nouvel évacuateur de crue du barrage de Gage II
}

\author{
Violaine DUGUÉ ${ }^{1}$, Michael PFISTER ${ }^{1}$, Fadi HACHEM ${ }^{1 *}$, Jean-Louis BOILLAT ${ }^{1 * *}$, Véronique NAGEL ${ }^{2}$, \\ Frédéric LAUGIER ${ }^{2}$
}

\author{
1. Laboratoire de Constructions Hydrauliques (LCH), Ecole Polytechnique Fédérale de Lausanne (EPFL), Station 18, CH-1015 Lausanne, Suisse \\ - e-mail : michael.pfister@epfl.ch \\ * Actuellement à STUCKY SA, Rue du Lac 33, CH-1020 Renens VD 1, Suisse \\ ** Actuellement expert indépendant, Chemin de la Brotte 4, CH-1163 Etoy, Suisse \\ 2. Electricité de France (EDF), Centre d'Ingénierie Hydraulique, Savoie Technolac, Le Bourget-du-Lac, France
}

\begin{abstract}
RÉSUMÉ. - Suite à des études hydrologiques récentes, le barrage de Gage II en France révèle un déficit de capacité d'évacuation des crues. Il a donc été décidé de construire un évacuateur de crue complémentaire en rive droite du barrage. Cet ouvrage est composé d'un PKWeir et d'un déversoir clapet, suivi d'une auge de réception et d'une galerie d'évacuation avec un déflecteur à sa sortie. L'association PKWeir-clapet permet de fixer deux cotes maximum admissibles, une pour la période froide et une autre pour la période chaude à un niveau supérieur. L'étude du comportement hydraulique de l'évacuateur a été réalisée sur un modèle physique à l'échelle $1 / 40$. Les résultats ont montré que l'association d'un PKWeir à 7 unités d'alvéoles avec un clapet de $27.4 \mathrm{~m}$ de largeur est suffisante pour évacuer la crue de projet. Plusieurs configurations de l'auge ont été testées pour minimiser le volume d'excavation tout en y optimisant les écoulements. Les mesures réalisées sur la galerie d'évacuation ont montré que celle-ci n'était pas mise en charge pour les débits considérés. Deux aérateurs ont été ajoutés en calotte de la galerie afin d'assurer la circulation d'air. A la sortie, un déflecteur a été installé pour allonger la trajectoire du jet jusqu'à son point d'impact. Un élargissement de la section a également permis d'augmenter la dispersion du jet.
\end{abstract}

Mots-clés : Evacuateur de crue, modèle physique, PKWeir, déversoir clapet, déflecteur

\section{Physical modeling of the new spillway of Gage II Dam}

\begin{abstract}
An updated hydrological study indicated that the existing flood spillway capacity of the Gage II Dam in France is deficient. The owner decided thus to build a new auxiliary spillway on the right side of the dam. This new spillway includes an intake that consists of a side channel combining a PKWeir and a flap gate weir, followed by a tunnel chute with a deflector at its end. The arrangement of the PKWeir and the flap gate weir allows fixing two different maximum water levels: A lower one during the cold period and an upper one during the warm period. To guarantee an adequate performance of this new spillway, a systematic hydraulic model investigation was performed on a physical model with a scale factor of 1/40. Results show that the combination of seven PKWeir units with a 27.4 m wide flap gate is optimal to evacuate the design flood. Several side channel configurations were tested to optimize the flow features and the excavation volume. Along the tunnel, free surface flow was observed for all discharges. Nevertheless, shock waves and the bend generate a highly rough flow surface, so that two aerators were added to insure free air circulation in the tunnel. A jet-generating deflector was installed at the downstream end of the tunnel to achieve a jet impact in the valley center.
\end{abstract}

Key-words: Spillway, physical scaled model, PKWeir, flap gate weir, deflector

\section{INTRODUCTION}

Le barrage de Gage II, exploité par Electricité de France (EDF), est situé sur la rivière Gage dans le département de l'Ardèche en France. Ce barrage en voûte mince, de $40 \mathrm{~m}$ de hauteur maximale sur fondation, possède un évacuateur de crue d'une capacité de $230 \mathrm{~m}^{3} / \mathrm{s}$ implanté sur la crête du barrage (Figure 1).

D'après une étude hydrologique récente, les nouvelles estimations de crues extrêmes sont supérieures à celles utilisées lors du dimensionnement de l'évacuateur existant. Par exemple, le débit de la crue de projet d'une période de retour de 1000 ans en période chaude est maintenant estimé à $675 \mathrm{~m}^{3} / \mathrm{s}$, soit trois fois plus que le débit de dimensionnement originel. Il est donc envisagé de construire un deuxième évacuateur de crue en rive droite du barrage, afin de limiter les impacts sur le barrage existant.

Le barrage voûte de Gage est sensible aux sollicitations thermiques, en particulier à cote haute en saison froide. Afin de limiter les sollicitations sur le barrage existant, EDF a fixé deux cotes maximales à ne pas dépasser, une pour la période froide (1009.00 NGF) et une autre pour la période chaude (1011.50 NGF) correspondant à la cote des plus hautes eaux (PHE) actuelle. Les altitudes sont exprimées en NGF Lallemand ; 1010.00 NGF Lallemand est noté (1010.00), par la suite.

Le nouvel évacuateur est dimensionné pour que l'aménagement global puisse évacuer la crue millénale en périodes 
a)

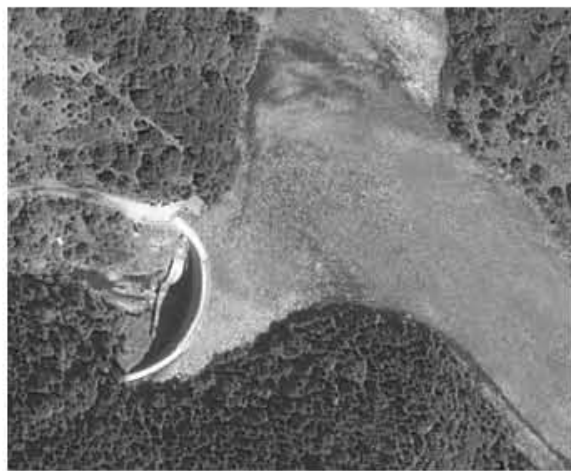

b)

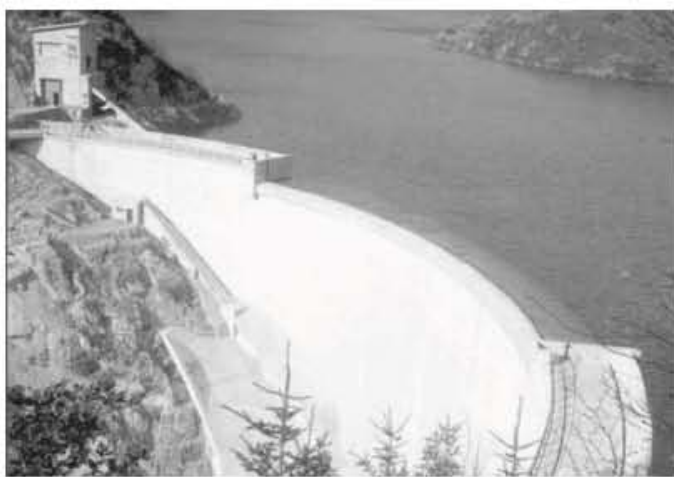

Figure 1 : (a) Vue aérienne du barrage et du réservoir de Gage II (Source : Google Earth) ; (b) photographie de la face aval du barrage et de l'évacuateur de crue existant (Source : EDF).

froide et chaude sans dépasser les cotes maximales définies sur la base du comportement du barrage (Tableau 1). Il se compose de l'amont vers l'aval des éléments suivants (Figure 2) :

- une prise de type PKWeir associée à un déversoir clapet ;

- une auge latérale de réception ;

- une galerie d'évacuation à surface libre de section en fer à cheval ;

- un déflecteur de sortie pour orienter le jet vers la rivière à l'aval du barrage.

En période chaude, les crêtes des deux parties de l'évacuateur se trouvent à l'altitude de retenue normale (1010.00). Le clapet, de $5 \mathrm{~m}$ de hauteur, est abaissé en période froide de sorte que la cote d'exploitation est alors imposée à (1005.00).

\section{ETUDE SUR MODÈLE REDUIT}

\section{II.1. Modèle physique}

Afin d'étudier en détail le comportement hydraulique complexe du nouvel évacuateur, un modèle réduit de l'aménagement à l'échelle $1 / 40$ a été construit au Laboratoire de Constructions Hydrauliques (LCH) de l'Ecole Polytechnique Fédérale de Lausanne (EPFL) [LCH-EPFL, 2011]. Le modèle est exploité en similitude de Froude et correspond à une surface de $160 \mathrm{~m}$ x160 $\mathrm{m}$ de la retenue, incluant la partie de rive où sera construit le nouvel évacuateur.

Le déversoir clapet, le PKWeir ainsi que l'auge latérale de réception ont été construits en PVC (Figure 3a). En effet, ce matériau respecte en similitude le coefficient de frottement du béton prévu sur prototype. La topographie du réservoir a

Tableau 1 : Conditions d'évacuation des crues de projet par l'ensemble des évacuateurs.

$\begin{array}{ccccccc}\begin{array}{c}\text { Période } \\ \text { de retour }\end{array} & \begin{array}{c}\text { Mois } \\ \text { de l'année }\end{array} & \text { Période } & \begin{array}{c}\text { Cote maximale } \\ \text { atteinte } \\ {[\mathbf{N G F}]}\end{array} & \begin{array}{c}\text { Débit total } \\ \text { à évacuer } \\ {\left[\mathbf{m}^{3} / \mathbf{s}\right]}\end{array} & \begin{array}{c}\text { Débit à évacuer } \\ \text { au barrage } \\ {\left[\mathbf{m}^{3} / \mathbf{s}\right]}\end{array} & \begin{array}{c}\text { Débit à évacuer } \\ \text { par le nouvel évacuateur } \\ {\left[\mathbf{m}^{3} / \mathbf{s}\right]}\end{array} \\ \mathrm{Q}_{1000} & \text { Avril-Novembre } & \text { chaude } & 1011.50 & 675 & 220 & 455 \\ \mathrm{Q}_{1000} & \text { Décembre-Mars } & \text { froide } & 1009.00 & 468 & 0 & 468\end{array}$

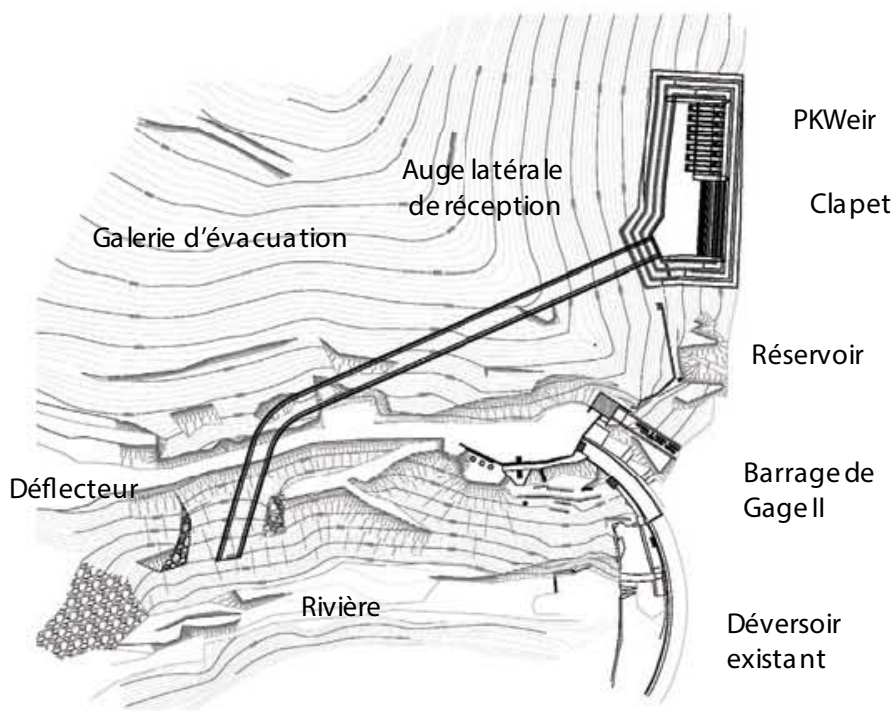

Figure 2 : Vue en plan du barrage de Gage II avec le nouvel évacuateur projeté. 
(a)

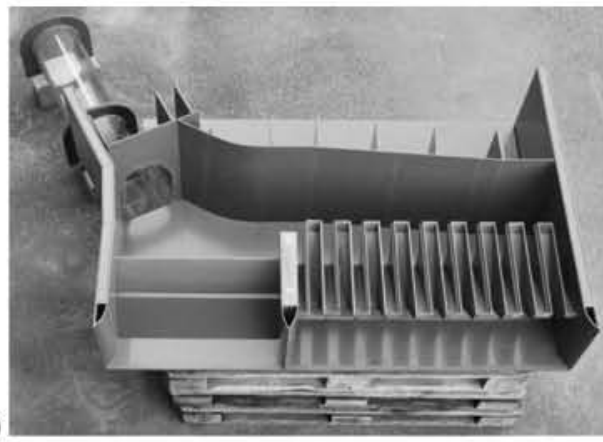

(b)

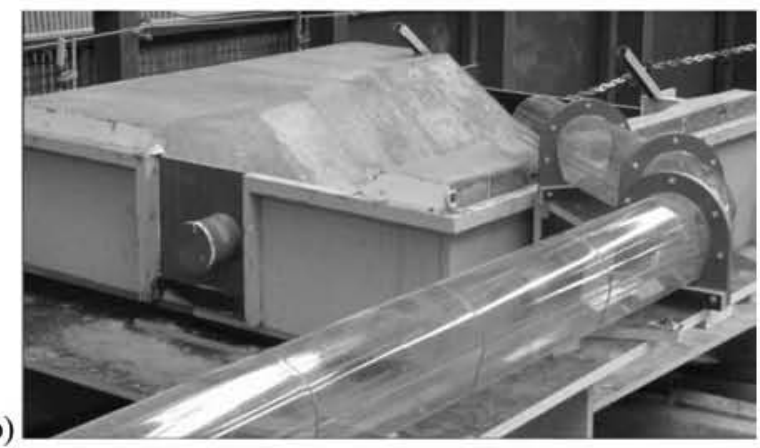

Figure 3 : (a) Maquette de la partie amont du nouvel évacuateur de crue, (b) Partie aval du modèle physique (galerie et zone de réception).

été reproduite à partir de plusieurs profils recouverts d'une couche de mortier. La galerie d'évacuation a été construite en PVC transparent afin de pouvoir visualiser les écoulements complexes tridimensionnels (Figure 3b). Une partie de la topographie aval a également été reproduite pour accueillir le jet issu de la galerie d'évacuation.

Le modèle est alimenté par une pompe dont le débit à l'entrée du modèle est mesuré par un débitmètre électromagnétique avec une précision de $1 \%$ du débit maximal. La hauteur d'eau dans la retenue est mesurée à l'aide de deux sondes à ultrasons d'une précision de $+/-1 \mathrm{~mm}$. Les hauteurs d'eau dans l'auge de réception sont estimées à l'aide de deux limnimètres situés sur les parois proches du PKWeir et du clapet. Les vitesses dans la galerie sont mesurées en plusieurs sections avec un micro-moulinet.

Des photographies zénithales permettent d'illustrer les écoulements à l'intérieur et en sortie de la galerie d'évacuation.

\section{II.2. Association PKWeir et Clapet}

La capacité de l'évacuateur a été évaluée pour trois configurations différentes de l'ouvrage d'entrée. Le nombre d'unités et la largeur du PKWeir ainsi que celle du déversoir clapet sont données dans le Tableau 2. La différence entre la Configuration 1 (Cam 1) et la Configuration 2 (Cam 2) repose exclusivement sur le nombre d'unités $N_{u}$ du
PKWeir. Dans les Configurations 2 et 3, l'évacuateur possède le même PKWeir mais une largeur de clapet différente. Les autres caractéristiques du PKWeir (largeur amont-aval de $13 \mathrm{~m}$, largeur d'une alvéole d'entrée de $1.6 \mathrm{~m}$, largeur d'une alvéole de sortie de $1.3 \mathrm{~m}$ et hauteur du parapet de $0.8 \mathrm{~m}$ ) sont conservées pour chacune des configurations et sont résumées en détail dans [Dugué et al., 2011]. Pour chaque configuration, les crues de projet de périodes chaude et froide ont été testées.

Les résultats expérimentaux de débitance de l'évacuateur sont représentés dans la Figure 4 pour les périodes chaude et froide.

- En période chaude (Figure 4a), la majorité du débit est évacuée par le PKWeir. La capacité d'évacuation de Cam 1 est supérieure à celles des deux autres (26\% supérieure à Cam 2 et $19 \%$ supérieure à Cam 3). En effet, le débit évacué par le PKWeir est proportionnel au nombre d'unités d'alvéoles. La comparaison entre Cam 2 et Cam 3 montre que la largeur du clapet a un impact mineur sur la capacité d'évacuation. Celle-ci est cependant de $6 \%$ supérieure pour Cam 3. On notera que pour $\mathrm{Q}_{1000}=675 \mathrm{~m}^{3} / \mathrm{s}$ dont $455 \mathrm{~m}^{3} / \mathrm{s}$ sont évacués par le nouvel évacuateur quel que soit le système retenu $\left(\mathrm{Cam} 1, \mathrm{Cam}^{2}\right.$ ou $\left.\mathrm{Cam}^{3}\right)$ sans dépasser la cote de la retenue pour les PHE à savoir (1011.50).

- La capacité hydraulique de l'évacuateur en période froide est représentée dans la Figure $4 \mathrm{~b}$. Pendant cette période, les

Tableau 2 : Configurations amont de l'évacuateur de crue testées.

Label
Configuration amont 1 (Cam 1)
Configuration amont 2 (Cam 2)

Configuration
amont 3
(Cam 3)

Schéma
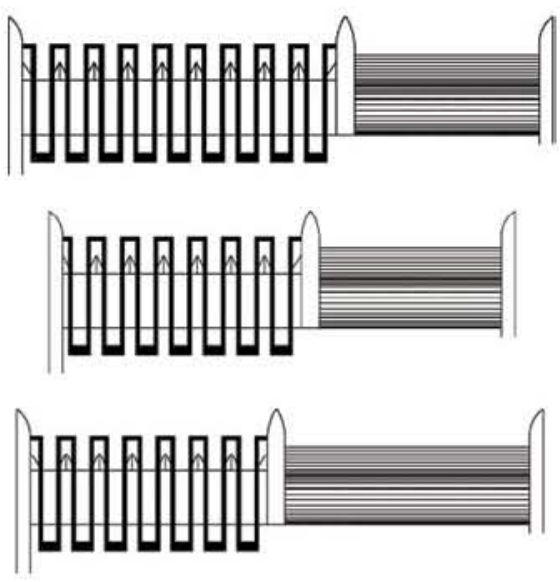

Largeur PKWeir

[m]

Largeur clapet

$\mathrm{N}_{\mathrm{u}}$
[unit]

9

34 

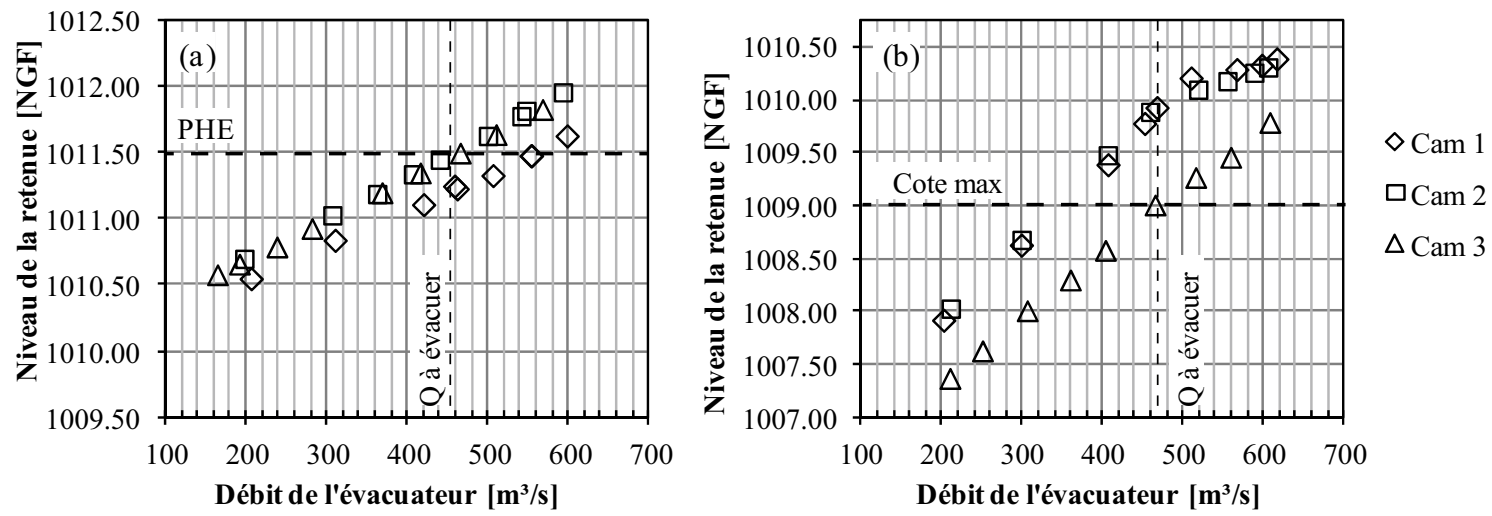

Figure 4 : Relations niveau-débit de l'évacuateur pour les trois différentes géométries (a) en période chaude avec le clapet en position relevée et (b) en période froide avec le clapet en position abaissée.

crues ne sont évacuées que par le clapet abaissé. Au cours de la phase expérimentale, la cote maximum admissible a été modifiée par EDF de (1010.00) à (1009.00) en période froide. Les résultats obtenus avec Cam 1 et Cam 2 satisfont donc l'ancienne condition à (1010.00) mais montrent un déficit de débitance pour la nouvelle condition à (1009.00). La nouvelle condition a pu être obtenue grâce à une augmentation de $7.4 \mathrm{~m}$ de la largeur déversante du clapet (Cam 3).

Les résultats obtenus pour les deux périodes montrent que Cam 3, avec un PKWeir de 7 unités d'alvéoles et une largeur de clapet de $27.4 \mathrm{~m}$, satisfait les conditions d'évacuation des deux crues millénales en périodes chaude et froide. Cette géométrie a été adoptée comme configuration de référence pour la suite des essais relatifs aux écoulements dans l'auge de réception.

\section{II.3. Ecoulement dans l'auge de réception}

Plusieurs géométries de l'auge de réception ont été testées sur le modèle physique, incluant l'inversion des positions respectives du PKWeir et du clapet, les pentes longitudinale et transversale de l'auge ainsi que sa largeur et sa profondeur [Dugué et al., 2011]. L'objectif était d'optimiser son fonctionnement hydraulique tout en minimisant les coûts d'excavation.
Les mesures ont été effectuées pour trois différentes altitudes du radier de l'auge en condition de période chaude, c'est à dire avec le clapet en position relevée. La configuration Cam 3 étudiée dans la section II.2 sert de référence avec un radier de l'auge fixé initialement à (998.00). Deux surélévations du radier de +2 et $+4 \mathrm{~m}$ ont été testées et comparées avec la configuration de référence. La débitance et le niveau d'eau mesuré dans l'auge près du PKWeir sont comparés dans la Figure 5. Les écoulements observés dans l'auge sont également illustrés par les photographies de la Figure 6.

Pour les faibles débits, la débitance de l'évacuateur est similaire dans les trois configurations (Figure 5a). La débitance n'est pas modifiée par la surélévation $+2 \mathrm{~m}$ du radier de l'auge dans la gamme des débits étudiés. En revanche, si le radier est surélevé de $+4 \mathrm{~m}$, la débitance est significativement diminuée pour les débits supérieurs à $400 \mathrm{~m}^{3} / \mathrm{s}$. A partir d'un débit compris entre 400 et $450 \mathrm{~m}^{3} / \mathrm{s}$, le niveau du réservoir augmente plus rapidement avec le débit que pour les autres configurations. Ceci est dû à un changement de régime d'écoulement dans l'auge de réception, passant d'un jet plongeant à un jet de surface [Rühli et al., 2010]. Cette transition met en évidence une capacité insuffisante de l'auge de réception, qui ne permet plus de satisfaire les conditions de passage d'une partie du débit $\left(455 \mathrm{~m}^{3} / \mathrm{s}\right)$ de la
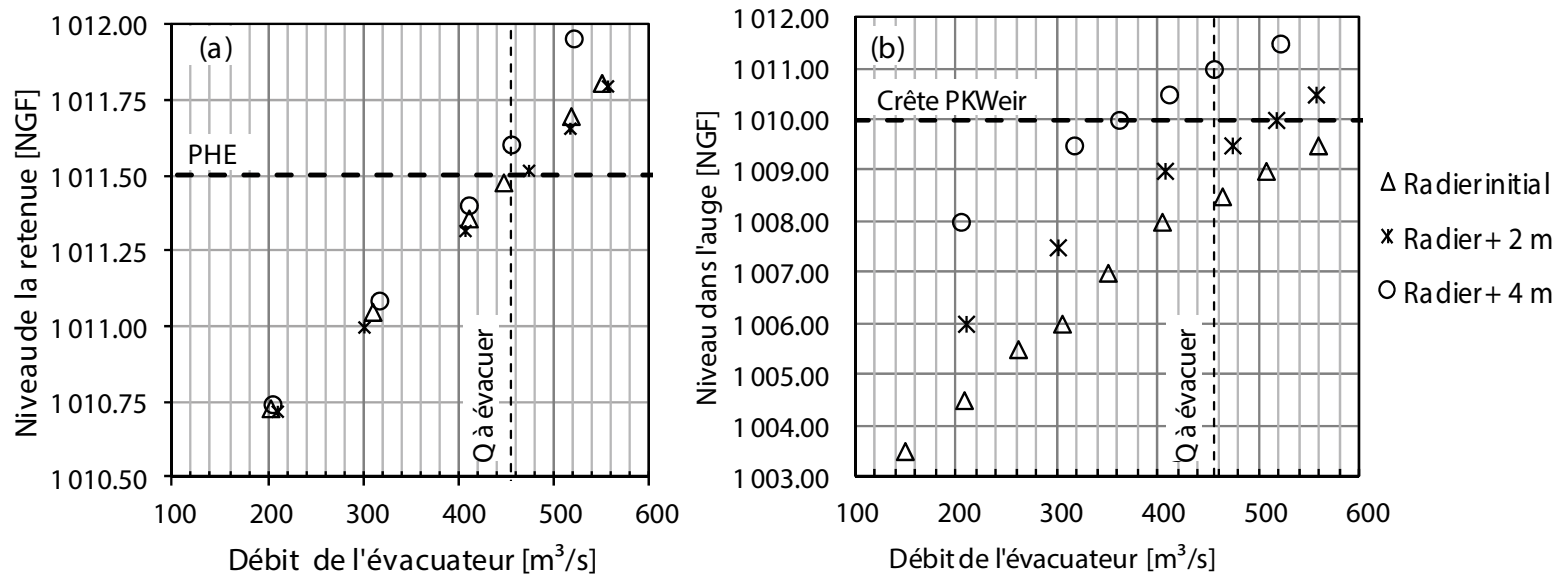

Figure 5 : Résultats obtenus en période chaude pour différents niveaux de radier de l'auge de réception (a) Relation niveaudébit de l'évacuateur et (b) Niveau d'eau dans l'auge de réception en fonction du débit. 
a)

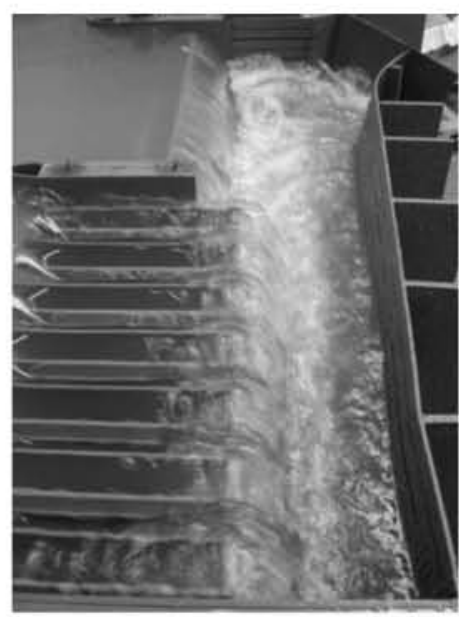

b)

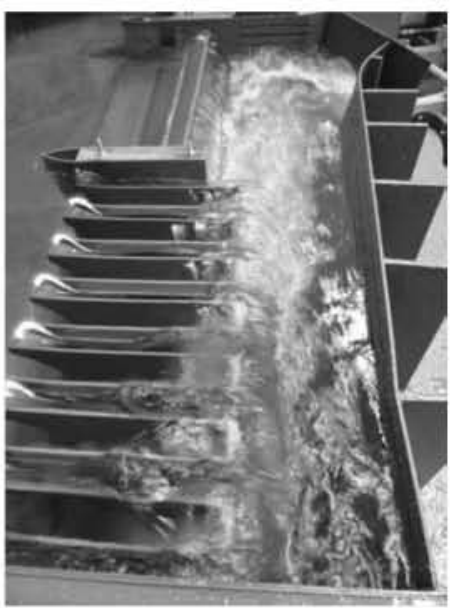

c)

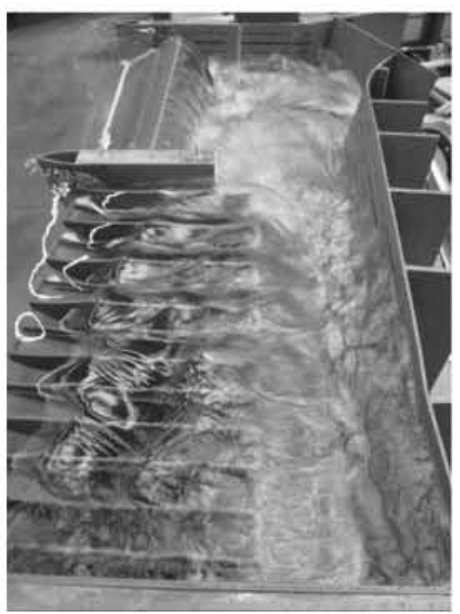

Figure 6 : Ecoulements dans l'auge pour la crue cinqmillénale en période chaude. Comparaison de l'influence de l'altitude du radier de l'auge: (a) Configuration de référence - Radier initial $\left(559 \mathrm{~m}^{3} / \mathrm{s}\right)$, (b) Radier $+2 \mathrm{~m}\left(557 \mathrm{~m}^{3} / \mathrm{s}\right)$ et (c) Radier $+4 \mathrm{~m}$ $\left(521 \mathrm{~m}^{3} / \mathrm{s}\right)$ - jet de surface.

crue millénale en période chaude. Il y donc lieu de ne pas excéder une surélévation du radier de plus de $2 \mathrm{~m}$.

La surélévation du radier, de $+2 \mathrm{~m}$ et $+4 \mathrm{~m}$ provoque une augmentation du niveau dans l'auge plus marquée du côté PKWeir que devant la galerie (Figures $5 \mathrm{~b}$ et 6 ). Le niveau d'eau dépasse la hauteur de crête du PKWeir (1010.00) provoquant son ennoiement partiel. Pour une surélévation de $+4 \mathrm{~m}$, cet ennoiement, initié à partir de $350 \mathrm{~m}^{3} / \mathrm{s}$ explique la perte de débitance du PKWeir.

Conformément aux prévisions, les différentes altitudes du radier ont un effet sur la débitance du nouvel évacuateur. Les deux critères à considérer pour le choix de la géométrie sont le respect des conditions de passage des crues de projet ainsi que le volume de roche à excaver. Finalement, le radier avec une surélévation de $+2 \mathrm{~m}$ par rapport à la configuration initiale s'est révélé comme la solution la plus adéquate (Figure 7). Finalement, afin d'éviter des interactions entre les écoulements provenant du PKWeir et la lame déversante du clapet en position relevée, ce dernier a été reculé de $3 \mathrm{~m}$ en direction de la retenue [Dugué et al., 2011].

\section{II.4. Galerie d'évacuation}

La galerie a été dimensionnée de manière à évacuer le débit de $610 \mathrm{~m}^{3} / \mathrm{s}$ sans qu'elle ne soit mise en charge. Par soucis de cavitation, un critère de vitesse d'écoulement maximale de $20 \mathrm{~m} / \mathrm{s}$ a été imposé. La pente longitudinale de la galerie est de $67 \%$ sur le tronçon amont et de $2 \%$ sur la partie courante (Figure 8).

Les différentes mesures réalisées dans la galerie ont permis de vérifier que celle-ci n'est pas mise en charge pour les débits à déverser par le nouvel évacuateur correspondants aux deux crues millénales [LCH-EPFL, 2011]. Pour les débits extrêmes cependant, l'écoulement touche la calotte sur une courte distance au début de la galerie ainsi que dans le coude en plan situé avant la sortie. Pour cette raison, deux aérateurs ont été ajoutés aux deux extrémités de la partie courante de la galerie, un après la rupture de pente et un avant le coude en plan (Figure 8). Ces deux aérateurs, modélisés par des trous en calotte de $0.8 \mathrm{~m}$ de diamètre, favorisent un écoulement à surface libre et absorbent les fluctuations de pression dans la galerie.
1012.00 NGF
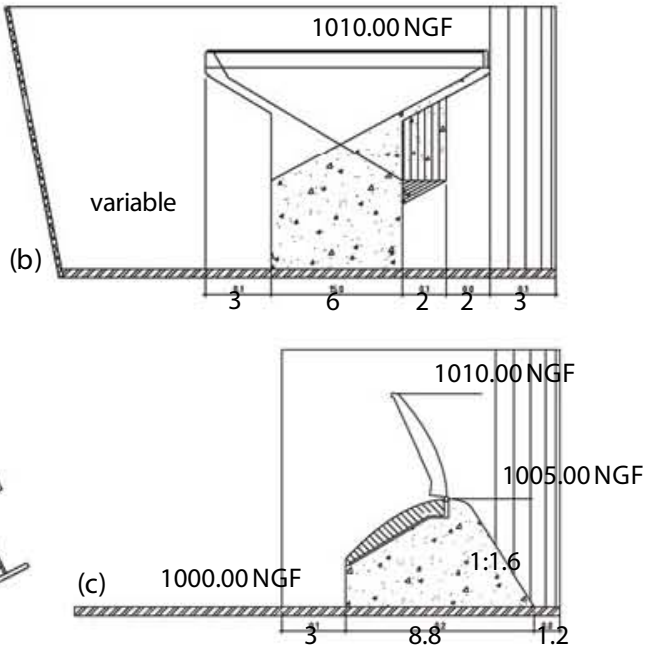

Figure 7 : Géométrie retenue pour la configuration amont de l'évacuateur. (a) Vue en plan), (b) coupe du PKWeir, (c) coupe du clapet. 


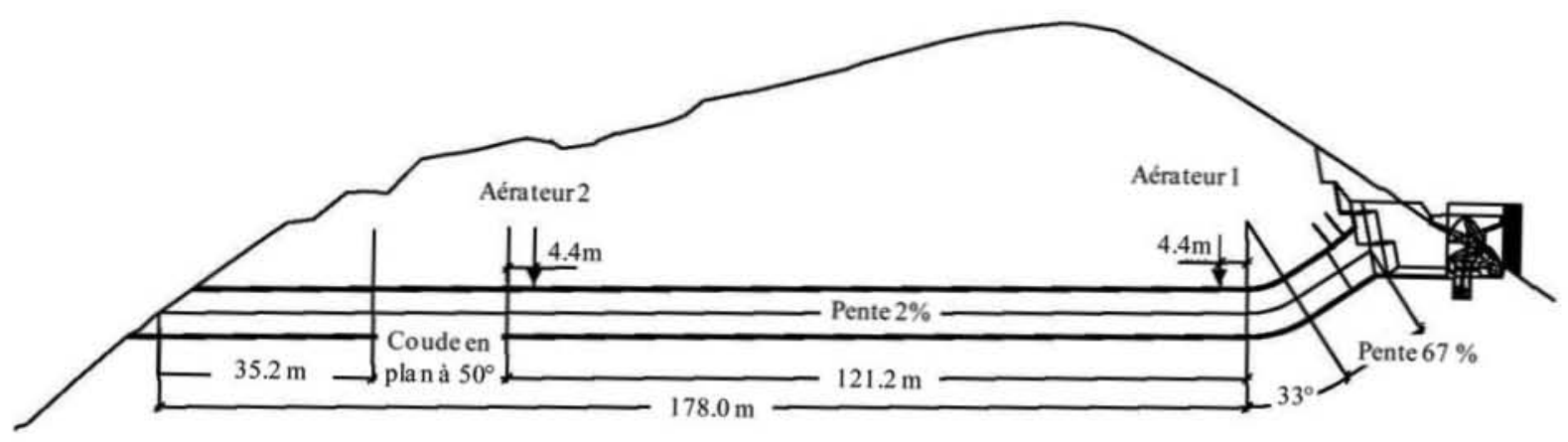

Figure 8 : Profil en long dans l'axe de la galerie d'évacuation.

Dans un premier temps, les hauteurs d'eau ont été modélisées numériquement avec le programme WS77 développé par l'USBR [Falvey, 1990]. Ce programme de modélisation 1D permet de simuler les écoulements dans les galeries à forte pente et d'étudier le risque de cavitation associé aux fortes vitesses. Les hauteurs d'eau mesurées sur le modèle physique sont systématiquement supérieures à celles modélisées numériquement, l'écoulement étant fortement aéré et tridimensionnel [LCH-EPFL, 2011]. Les valeurs mesurées sur le modèle physique ont été retenues car les phénomènes mis en jeu y sont mieux représentés. Les vitesses mesurées dans la section courante de la galerie sont comprises entre 8 et $16 \mathrm{~m} / \mathrm{s}$ à l'échelle prototype. La valeur limite considérée pour le risque de cavitation, fixée à $20 \mathrm{~m} / \mathrm{s}$, n'est donc pas atteinte. L'évolution de l'écoulement torrentiel dans le coude est illustrée dans la Figure 9. Pour les faibles débits compris entre 200 et $300 \mathrm{~m}^{3} / \mathrm{s}$ (Figure 9a), l'écoulement subit un balancement qui le conduit en calotte. Le début de l'onde de choc stationnaire, résultant du changement de direction de l'écoulement torrentiel [Reinauer, Hager, 1997], est visible sur le côté droit de la galerie. Pour les débits plus élevés (Figure 9b), le balancement de l'écoulement initié à l'entrée du coude conduit à un retournement complet de l'écoulement qui adhère à la paroi de la galerie. L'écoulement est alors fortement émulsionné dans la dernière section de la galerie.

\section{II.5. Déflecteur}

A la sortie de la galerie d'évacuation, l'eau doit être réceptionnée dans la partie aval de la rivière, à l'endroit où la section est la plus large et où un petit bassin s'est formé. Plusieurs géométries de sortie ont été testées sur le modèle dont deux sont présentées dans cet article. La configuration aval 1 correspond à une sortie sans aménagement particulier, adaptée à la topographie de sortie et conservant une pente longitudinale de $2 \%$. La configuration aval 2 possède un déflecteur linéaire vertical (Rapport Hauteur/Longueur de 1/5) placé en bordure de la topographie, non perpendiculairement à l'axe de la galerie. Un élargissement a également été introduit en rive droite (voile de $12^{\circ}$ ) afin d'augmenter la dissipation du jet.

L'estimation du point d'impact de la trajectoire supérieure du jet est obtenue à l'aide d'une grille tracée sur la topographie aval. Chaque carreau couvre une surface prototype de $4 \mathrm{~m} \times 4 \mathrm{~m}$. Des photographies zénithales ont été prises pour les différents débits et types de déflecteurs afin de visualiser le point d'impact, la largeur et la forme du jet. Un exemple de résultats obtenus pour la crue cinqmillénale en période chaude est présenté dans la Figure 10.

Dans la Configuration aval 1, la distance du point d'impact est inférieure à celle de la Configuration aval 2. Cette dernière, du fait de l'élargissement de la section de sortie, conduit à une expansion latérale du jet plus importante et donc à une meilleure dissipation.

Les points d'impact dans l'axe du jet, obtenus d'après les photos zénithales, sont comparés pour les deux configurations présentées ici (Figure 11). La zone approximative de réception souhaitée (bassin existant au fond de la vallée) est également représentée sur la figure. Le déflecteur de la Configuration aval 2 augmente sensiblement la distance du point d'impact par rapport aux résultats de la Configuration (a)

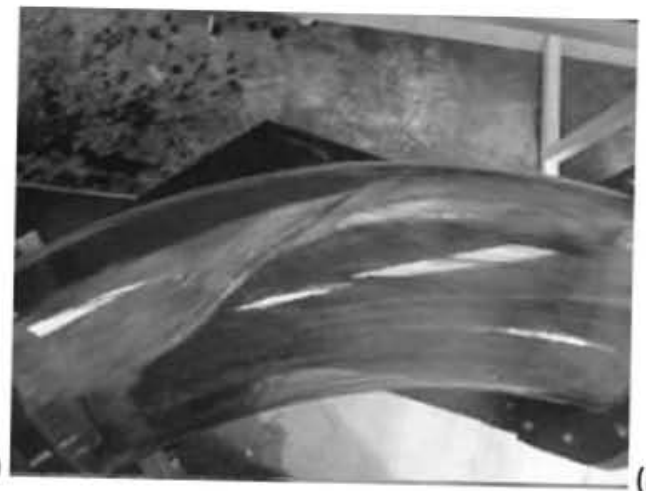

(b)

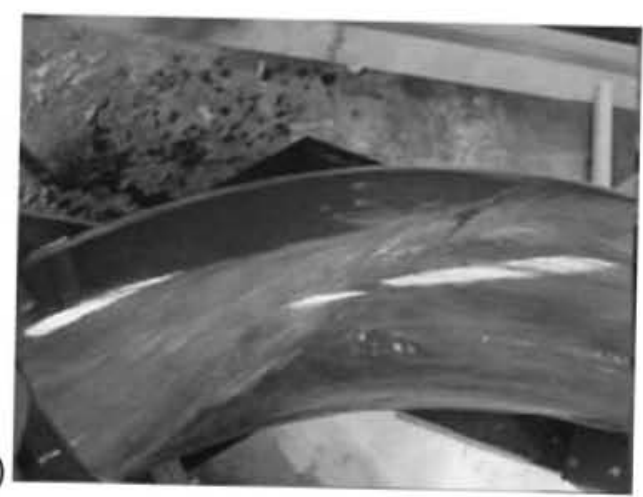

Figure 9 : Vue zénithale des écoulements dans le coude en plan, de droite à gauche. (a) $303 \mathrm{~m}^{3} / \mathrm{s}$ et (b) $420 \mathrm{~m}^{3} / \mathrm{s}$. 
(a)

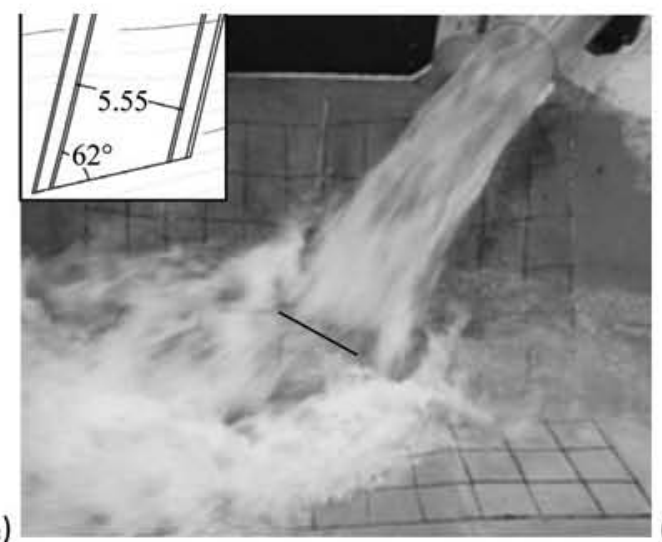

(b)

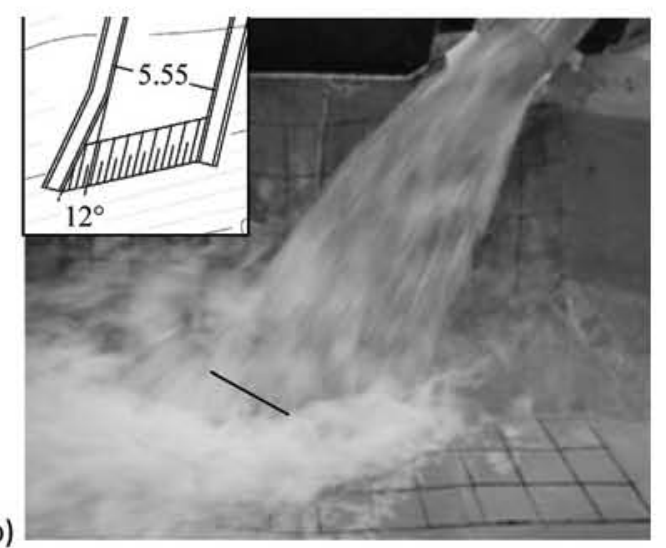

Figure 10 : Position du point d'impact et expansion du jet pour un débit de $563 \mathrm{~m}^{3} / \mathrm{s}$ pour (a) la Configuration aval 1 et (b) la Configuration aval 2. La ligne noire indique la position du point d'impact.

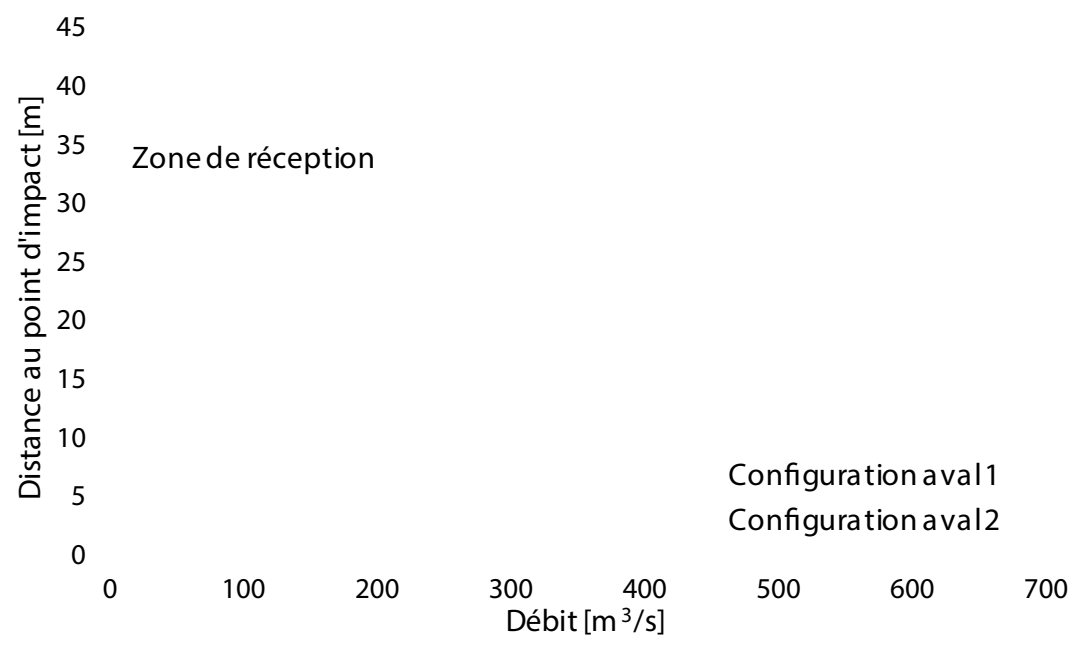

Figure 11 : Distance axiale entre la sortie de la galerie et le point d'impact de la trajectoire supérieure du jet pour les deux configurations étudiées.

aval 1 et fait atterrir le jet dans la zone de réception souhaitée pour les débits les plus importants. De plus, le jet est plus large avec la Configuration aval 2, impliquant une densité d'énergie réduite.

\section{CONCLUSIONS}

Un nouvel évacuateur de crue, composé d'un PKWeir et d'un déversoir à clapet suivis d'une auge réceptrice, a été choisi pour augmenter la débitance du barrage de Gage II. Cette réhabilitation est imposée par la définition de nouvelles crues de projet, trois fois supérieures à celles adoptées lors du dimensionnement initial. Les principaux objectifs de la modélisation physique du nouvel évacuateur à l'échelle $1 / 40$ portaient sur le respect des conditions de passage des crues de projet, l'optimisation des écoulements dans l'auge de réception tout en limitant les frais d'excavation, les écoulements dans la galerie d'évacuation et enfin la redirection du jet vers la zone d'impact prévue dans le lit du cours d'eau. Les conclusions suivantes ont pu être formulées :

- La configuration permettant de satisfaire les conditions de passage des crues de projet associe un PKWeir de $26.6 \mathrm{~m}$ de large composé de sept unités d'alvéoles et un déversoir clapet de $27.4 \mathrm{~m}$ de largeur.

- Les essais d'optimisation de l'auge de réception ont démontré l'influence de sa géométrie sur la débitance du PKWeir. Plusieurs configurations ont été testées en faisant varier l'altitude du radier, la pente du fond ainsi que la largeur de l'auge. La solution retenue est caractérisée par un radier surélevé de $2 \mathrm{~m}$ par rapport au dimensionnement initial, soit $10.0 \mathrm{~m}$ au dessous du niveau de crête du PKWeir.

- Les mesures effectuées dans la galerie d'évacuation ont permis de vérifier qu'elle n'était pas mise en charge dans la gamme des débits considérés et que les vitesses maximales ne dépassaient pas la valeur limite imposée par le risque de cavitation. Cependant, deux aérateurs ont été installés en calotte afin de faciliter la circulation d'air dans la partie supérieure de la galerie.

- Les études menées sur la sortie de la galerie ont montré que la mise en place d'un déflecteur permet d'ajuster la longueur de trajectoire du jet. Un élargissement de la section de sortie en rive droite (avec un voile de $12^{\circ}$ ) permet d'augmenter efficacement l'expansion latérale du jet ainsi que la dissipation d'énergie avant l'impact. 


\section{REFERENCES ET CITATIONS}

Dugue V., Hachem F., Boillat, J-L., Nagel V., Roca J.-P., \& LAUGIER F. (2011) - PKWeir and flap gate spillway for the Gage II Dam. Proc. Intl. Workshop. Labyrinth and Piano Key Weirs CRC Press, Taylor \& Francis Group, ISBN 978-0-41568282-4. 35-42

EpfL-Lch (2011) - Aménagement de Montpezat - Barrage de Gage II. Rapport final d'étude $L C H N^{\circ} 03 / 2011$, non publié
Falvey H. T. (1990) - Cavitation in Chutes and Spillways. USBR Engrg. Monograph Denver, Colorado, USA. 42

Reinauer R., \& Hager W.H. (1997) - Supercritical bend flow. J. Hydraulic Eng. 123(3) : 208-218

RÜHLI E., Pfister M., \& LAIS A. (2010) - Modelluntersuchungen zum Einlaufbauwerk und zur Kapazität des HochwasserEntlastungsstollens in Lyss. Wasser Energie Luft. 102(4) : 281-288 